\title{
The economic relationships of essential oil production as a basis for supply chain modeling
}

\author{
R.R. Timirgaleeva ${ }^{1,2,}{ }^{*}, M . V$. Verdysh ${ }^{2}, A . A$. Popova $^{2}$, and N.Yu. Polyakova ${ }^{2}$ \\ ${ }^{1}$ V.I. Vernadsky Crimean Federal University, 4, Vernadsky prosp., Simferopol, 295007, Russian \\ Federation \\ ${ }^{2}$ Research Institute of Agriculture of Crimea, 150, Kievskaya str., Simferopol, 295493, Russian \\ Federation
}

\begin{abstract}
This article reveals that essential oil production is a complex system with a large number of economic ties arising in the process of growing and processing as well as distribution of products made from essential oils raw materials. Multileveled manufacturing process of essential oils products requires coordination of all the elements to reduce costs in the entire supply chain. It is proposed to use mathematical modeling of the supply chain based on the method of main faces, which allows to take into account the specifics of the problem and makes it possible to use a variety of combinatorial and heuristic algorithms.
\end{abstract}

\section{Introduction}

During the production and consumption of any type of product between the participants in the process, numerous connections arise through which the economic interests of producers and consumers are realized [1]. The system of economic relations can be considered as a set of organizational and economic measures that provide the participants in the production process with the necessary conditions for their development, proportionality, rhythm and optimal operation of all links of a single technological chain, as well as timely promotion and sale of the final product to the consumer [2]. The mechanism of relationships consists of many elements, which are combined into three groups: organizational and management, production and technology, and financial and economic relationships. These groups of relationships cover all stages of production and sale of the final product [3].

Production and technological relations directly affect production and manifested through the division of labor and material resources in the implementation of the technological production process. Financial and economic relations reflect the essence of commodity-money relations through such economic categories as cost, price, profit, profitability. In this case, a special meaning justified to distribution of profits is of particular importance. The main task of financial and economic relations is to ensure an equivalent exchange of labor results between all participants of production activities [4].

\footnotetext{
* Corresponding author: renatimir@gmail.com
} 
One of the branch of production, characterized by a significant number of different connections, is the production of essential oil products - essential oils, plant extracts, hydrolates.

\section{Materials and methods}

The theoretical and methodological basis of the study was the fundamental provisions of logistics, legislative and regulatory acts regulating essential oil production, monographs and scientific publications of domestic and foreign scientists. General scientific and special research methods, methods of systematization and modeling were used.

\section{Results}

Essential oil products are characterized by a wide range of applications. In the Russian Federation, the main consumer of essential oil products is the perfumery and cosmetics industry, which occupies 3,5\% of the volume of chemical production in Russia, and $0,1 \%$ of the gross domestic product. This is due to the fact, that essential oils are an important ingredient in the production of various types of perfumery products and the increase in their production is aimed at implementing the state policy of import substitution. Essential oil products are also used in pharmaceutical, chemical and some other industries [5].

As of 2020, 55,1\% of all areas of essential plants in the Russian Federation were located in the Republic of Crimea. The region produces a significant share of Russian essential oils and other products. A specific feature of essential oil production in the Republic of Crimea is the prevalence of flower and herbaceous raw materials in the structure of the gross collection of essential oils [6]. The main source of essential oils is the overhead part of most of the traditional (lavender angustifolia, clary sage, essential oil rose) and promising (oregano, thyme, medicinal sage, hyssop, tauric wormwood and others) essential plants. A feature of these types of raw materials is a fairly high moisture content and the content of essential oils in external containers, which results in their losses during processing delays and disruptions in the technological process [7]. These features do not allow considering the production of essential oil raw materials in isolation from its processing. In addition, coriander, which is an essential oil crop, occupies significant areas in Crimea, but most of the crop, which is not processed and exported as raw material to the countries of Southeast Asia, where it is used as a spice.

Taking into account the multistage process of production of products, which in turn is a raw material for other industries, it is possible to characterize essential oil production as a whole, as a set of a number of interconnected industries, the result of which is the production of essential oil and other types of products.

We believe that the assertion given by the author of work [8] regarding the fact that essential oil production is a complex complex, including three sectors: agricultural, industrial and marketing, is completely justified (Figure 1).

Let's take a closer look at the functions of each sector. Thus, the function of the agricultural sector is the production of essential oil raw materials through the cultivation and collection of cultivated and collection of wild essential oil plants. Essential oil raw materials are produced by commodity producers of various organizational forms and forms of ownership. Depending on the volume of production, type of raw materials, specialization and location, commodity producers can be represented by agricultural holdings, specialized agricultural organizations, farms, procurement enterprises, as well as individuals. 


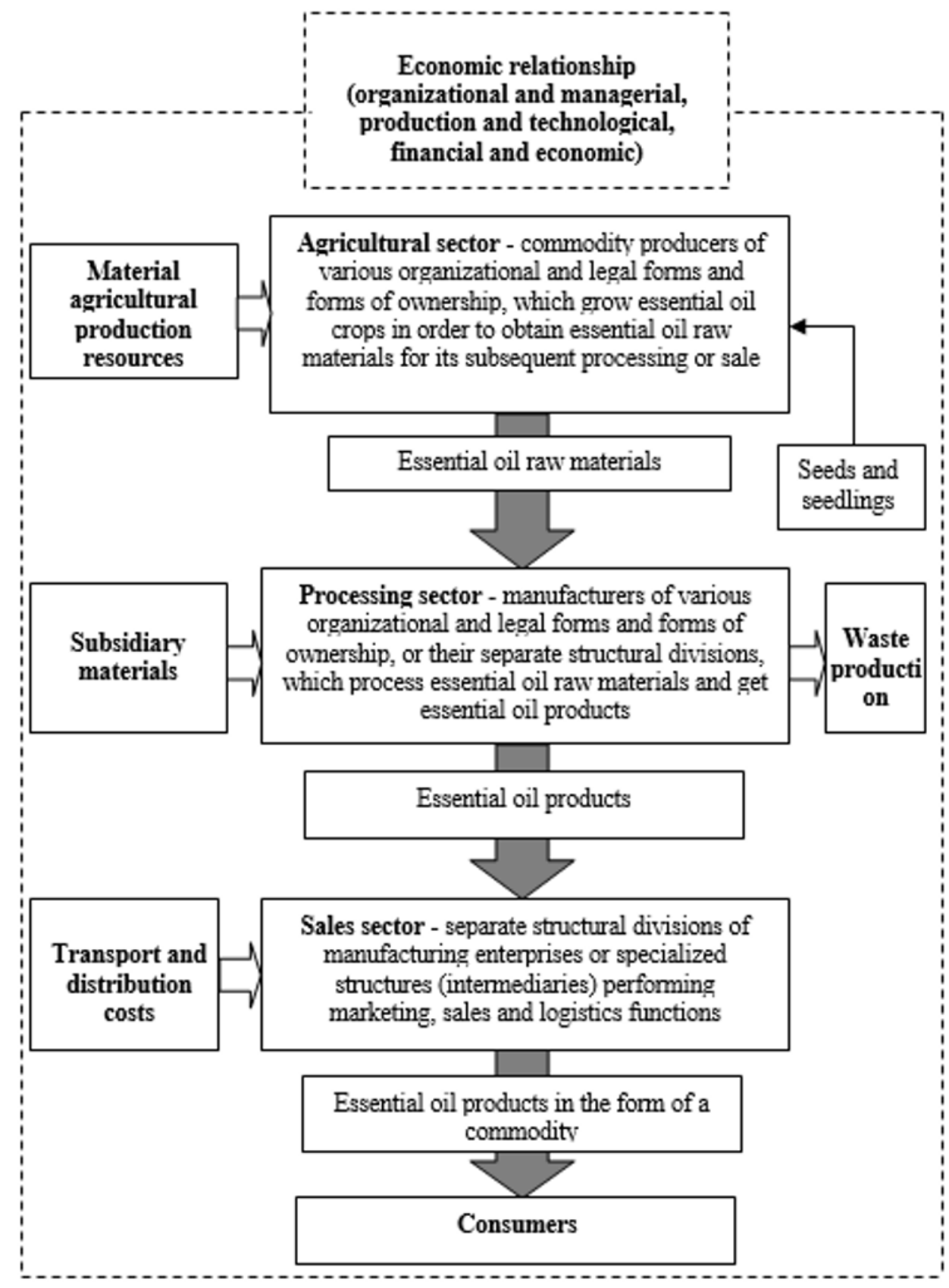

Fig. 1. The scheme of the essential oil complex (compiled according to [8] with author's additions).

The industrial sector includes facilities for the processing of essential oil raw materials, packaging and storage of the resulting essential oil products, which are used in other industries and in medicine. Enterprises that produce essential oil raw materials, especially herbaceous and floral ones, which have a limited processing time, can simultaneously process them. In a market economy, foreign trade restrictions, as well as increased competition with foreign producers of essential oils and synthetic fragrances, the role of the 
sales segment of essential oil production is increasing, which is the link between the production of essential oil products and its consumers.

Technologically, in accordance with the functions of the sectors of the essential oil complex, the process of producing essential oil products consists of four main stages:

- growing of essential oil raw materials;

- harvesting, transportation and acceptance of essential oil raw materials;

- storage of essential oil raw materials (impossible for herbaceous and floral raw materials of some essential plants);

- industrial processing of essential oil raw materials.

From the point of view of economic processes, essential oil production is a system of economic relations in the process of growing, processing essential oil raw materials and includes an agricultural complex that produces agricultural products, as well as a processing complex that processes raw materials, production of essential oil products.

The above biological features of ether plants, as well as the technology of their cultivation and the multistage production of essential oil products dictate the need to coordinate the actions of all links of production: harvesting, transportation, timely processing. At the same time, such important organizational issues as the timely conclusion of contracts for the supply of raw materials, the availability in sufficient quantities for certain short periods of labor, transport, energy resources, processing line capacities are also being resolved.

\section{Discussion}

Separately, we note the growing interest in essential oil products from European countries. This interest is manifested in an increase in demand for essential oils, which European manufacturers use for the production of a wide range of food products and beverages, as well as in medicine $[9,10]$. The increasing demand for essential oils is forcing European importers to look for new sources. The European market is ready to consume at least $30 \%$ of the total essential oils market today. At the same time, as studies have shown, essential oils go through many distribution channels before reaching the end consumer. Due to the logistics services of importers and suppliers, the costs associated with transportation, storage, loading and unloading operations increase, which ultimately affects the cost of the final product [11]. These aspects orient importers to import essential oils from countries of origin. In such conditions, it is necessary to strengthen control of supplies, which will ensure their sustainability, especially when supplying strategically important essential ingredients.

In order to avoid the monopoly influence of one supplier, it is necessary to organize supplies from various sources, as well as develop inventory management issues that will ensure the uninterrupted operation of enterprises - consumers of essential oil raw materials, as well as increase the reliability of supply and the sustainability of all business processes.

For the formation of effective interaction between the subjects of essential oil production, it is necessary to study the costs of the supply of raw materials associated with transportation, storage, preconditioning and other logistics operations that form supply chains and are used to determine the location of factories producing products from essential oil raw materials [12]. At the same time, the supply chain should be formed taking into account the main rule of logistics - rule $6 \mathrm{H}$ (to produce and distribute a product in the right quantity and quality, at the right time, in the right place with the lowest cost). An important condition is the management of quality improvement processes, which requires an analysis of the entire chain as a holistic system in which all stakeholders are linked to produce highquality products at all levels - local, national, international. 
An effective tool for the implementation of this task is the mathematical modeling of the supply chain, based on the establishment of the interaction of all stages of the essential oil production process, starting with business processes in the agricultural sector and pumping to final consumption. The main problem is the presence of many variables, which makes the model quite complex.

The existing methods of linear optimization, based on various modifications of the simplex method, do not allow solving practical problems of optimizing economic systems, which include the logistics system of essential oil production, since the dimension of these problems exceeds several hundred variables and constraints.

At present, methods based on various modifications of the interior point method are used to solve such problems. We propose the method of principal faces, which can be applied to construct an effective heuristic algorithm for finding integer solutions to the linear optimization problem. If the optimal vertex obtained by any method is known, then the main constraints - inequalities - can be distinguished and, taking them into account, the linear optimization problem is solved anew by the method of principal hyperplanes.

When calculating at each iteration, the systems of inequalities will specify kdimensional $(k=n-1, n-2, \ldots 1)$ cones obtained by successive elimination of the main edges. In the vicinity of the optimal vertex, the cones define k-dimensional boundary regions (k-sided angles) whose linear hulls have a minimum distance from the target hyperplane. Since the cones are projected onto the coordinate subspaces $R_{k}$ of the same dimension, it is easy to find admissible integer points near the optimal vertex in the reverse order of iterations. Fixing the found values of the variables, at each iteration, new integer coordinates are calculated, and in $n$ iterations a fairly good integer solution can be obtained.

Simple heuristic method for choosing the main facets with a fairly effective elimination of unnecessary constraints is to go over to a linear optimization problem with a system of homogeneous inequalities $\left(\bar{n}_{i} X\right)-b_{i} x_{n+1} \leq 0, X \geq 0,0 \leq x_{n+1} \leq 1$ constraints in the space $R_{n}+1$. The original polyhedron is converted to a pyramid. The pyramid is the convex hull of the original polytope lying in the hyperplane $x_{n+1}=1$ and being the base of the pyramid, as well as the point $\varnothing$ outside this hyperplane, called the apex, $\varnothing$-zero in $R_{n}+$ 1.

The side faces of the pyramid are the convex hulls of the faces of the polyhedron and zero, their adjacency matrix is equivalent to the adjacency matrix of the original polytope.

The objective function of the pyramid is determined from the conditions:

1) the optimal vertices of the pyramid and polyhedron must coincide;

2) the main facets at all iterations, except for the last one, should be the side faces of the pyramid (the choice of the base reduces the problem to the original one).

These conditions are met by the objective function, which is the convex hull of the optimal hyperplane $\mathrm{z}$ in the displaced subspace $x_{n+1}=1$ and zero; it is determined by the formula $\tilde{z}=(\bar{n} \cdot X)-z^{*} \cdot x_{n+1}$, where $z^{*}=\max z$.

Optimal value $\tilde{z}^{*}=0$. Since the quantity $z^{*}$ is not known in advance, to fulfill the conditions, a transition is made to the combined (direct and dual) problem. The problem is formulated in matrix form (X, Y, C, B are column vectors):

find max $\mathrm{F}=\mathrm{CTX}-\mathrm{BTY}$ subject to the constraints $\mathrm{AX} \leq \mathrm{B},-\mathrm{ATY} \leq-\mathrm{C}, \mathrm{X} \geq 0, \mathrm{Y} \geq 0$.

The solution to the problem will be the point $\left(X^{*}, Y^{*}\right)$ where $X^{*}, Y^{*}-$ the solution of direct and dual problems; the optimal value of $\mathrm{F}$ is zero.

Similarly to this problem, an equivalent with an additional coordinate $\mathrm{w}$ is formulated: find $\max \tilde{F}=-q^{T} U$ under the constraints $\tilde{A} U-q w \leq 0 ; U \geq 0, w \geq 0, w \leq 1$, where $U=(X, Y)^{T} ; q=(-C, B)^{T} ; \tilde{A}=\left(\begin{array}{cc}0 & -A^{T} \\ A & 0\end{array}\right)$, and the optimal value $\tilde{F}=0$.

Since the optimal target hyperplane touches this pyramid along the edge $\left(\emptyset ;\left(X^{*}, Y^{*}, 1\right)\right)$, the second condition will be satisfied if the choice of the base $\mathrm{w}=1$ is 
ignored. The solution of this problem by the method of faces is equivalent to solving the original problem with a system of homogeneous inequalities in the space $\mathrm{R}_{\mathrm{n}}+1$ with the exception of the choice of the base $x_{n+1}=1$ however, the a priori value of the value $\max \widetilde{F}=0$ gives additional opportunities to filter out unnecessary constraints. In the case of large dimensions, it is expedient to solve this problem using a partial adjacency matrix determined from the polar polyhedron.

\section{Conclusion}

The study showed that essential oil production is a complex system with a large number of economic relations, formed as a result of the interaction of the agricultural, industrial and marketing sectors, each of which performs its functions separately. This approach leads to unreasonable costs in the supply chain and cannot ensure efficient production and distribution of essential oil products. One of the tools for solving this problem is the mathematical modeling of the supply chain. Considering the presence of many variables, the model turns out to be such difficult. To solve the problem of optimization of a complex economic system, which is the system of essential oil production, the method of main faces is proposed. On the basis of this method, an effective heuristic algorithm for finding integer solutions to the linear optimization problem, which is the main task of logistics, is constructed. Its solution is aimed at reducing costs and increasing the efficiency of the entire system of essential oil production. It should be noted that when solving practical problems, the method of edges is more effective than others, since it takes into account the specifics of the problem, which is not done when using known methods.

A geometric approach to solving linear optimization problems should stimulate research in n-dimensional geometry on such issues as the adjacency of facets, isomorphism of polyhedra, which were not previously in demand. In addition, this approach opened up the possibility of using a variety of combinatorial and heuristic algorithms. The main problem of this method is to determine the compatibility of the system of constraints. Its solution is the task of our next research.

\section{References}

1. Vidyapin V.I., Zhuravleva G.P., Gulyamova S.S., Sharifkhodzhaev M.Sh. Economic theory. - Tashkent, 1999, 449 p.

2. Sartova R.B. Improvement of economic relations of agricultural producers in the field of processing, marketing of agricultural products. - Pavlodar: Kereku, 2015, 146 p.

3. Sidorov V.A., Ampar L.G. The category of production relations as the basis of the theory of social production, Theory and practice of social development, 2013, No. 11, P.455-458

4. Okladchik S.A. Development of organizational and economic relationships in agroindustrial integration // Actual problems of economics and law, 2016, V.10, No. 3. P.28-38

5. Draft Strategy for the development of the perfumery and cosmetic industry in Russia until 2030 "[Electronic resource]. - URL: https: //gmpnews.ru/wpntent/uploads/2018/10/Strategia_razvitia_PKP.docx

6. Federal State Statistics Service [Electronic resource] - URL: http://www.gks.ru

7. Nazarenko L.G., Afonin A.V. Ethernos of the south of Ukraine, Simferopol: Tavria, 2008, 144 p. 
8. Cherkashina E.V. Economics and organization of rational use and protection of lands of the essential oil and medicinal industry in the Russian Federation: dis. ... doc. econom. Sciences: 08.00.05 / E.V. Cherkashin; State University of Land Management. M., 2014, 419 p.Worwood Valerie Ann. The Complete Book of Essential Oils and Aromatherapy - New World Library, 1991, 448 p.

9. Peters M. Essential oils: historical significance, chemical composition and medicinal uses and benefits. - Nova Science Pub Inc, 2016. - 201 p.

10. Martin Ch. Logistics and Supply Chain Management.4th Edition. - Pearson Education, 2011. - 288 p.

11. Waters D. Logistics: An Introduction to Supply Chain Management Palgrave Macmillan, 2003. - 364 p. 